\title{
Producción primaria y eficiencia fotosintética en Cuenca Alfonso, Bahía de La Paz, Golfo de California, México
}

Primary production and photosynthetic efficiency in Alfonso Basin, La Paz Bay, Gulf of California, Mexico

\author{
Gerardo Verdugo-Díaz ${ }^{1}$, Aida Martínez-López¹, María Magdalena \\ Villegas-Aguilera ${ }^{1}$ y Gilberto Gaxiola-Castro ${ }^{2}$
}

\begin{abstract}
'Instituto Politécnico Nacional, Centro Interdisciplinario de Ciencias Marinas, (CICIMAR-IPN), Departamento de Plancton y Ecología Marina, Apdo. Postal 592, Av. Instituto Politécnico Nacional S/N Colonia Playa Palo de Santa Rita, La Paz, BCS, CP 23096, México. gverdugo@ipn.mx

${ }^{2}$ Centro de Investigación Científica y de Educación Superior de Ensenada, División de Oceanología, Carretera Ensenada-Tijuana No. 3918, Zona Playitas, CP 22860, Ensenada Baja California, México

Abstract.- Quantification of photosynthetic efficiency and productive capacity of the phytoplankton community was the objective of the surveys that were conducted in December 2005, May and August 2006; and February, May and November 2007 in Bahía de La Paz (Cuenca Alfonso). Samples of water were collected from 7 levels of surface irradiance (100, 55, 33, 10, $3,1$ and $0.1 \%)$, of the euphotic zone $\left(Z_{\text {eu }}\right)$. In situ primary production estimations were performed by $14 \mathrm{C}$ assimilation methodology. There were 2 basic assimilation ratios patterns; one with subsurface maxima during February and May 2007 and other with a tendency of increasing values as the lower limit of the $Z_{\text {eu }}$ was approached (December 2005; May and August

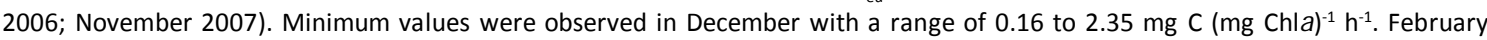

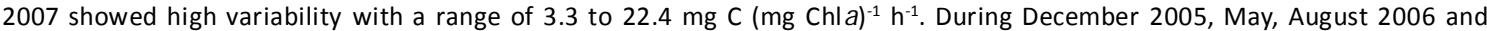
November 2007, there was an increase to $0.1 \%$ of surface irradiance, which reached $10.22 \mathrm{mg} \mathrm{C}(\mathrm{mg} \mathrm{Chla})^{-1} \mathrm{~h}^{-1}$ in August 2006.

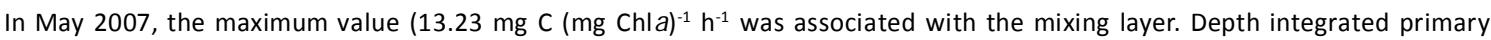
production (PP) value up to $350 \mathrm{~g} \mathrm{C} \mathrm{m}^{-2}$ year $^{-1}$ point out the high productive feature of the study site in Bahía de La Paz. A significant increase in the PP to consider $0.1 \%$ of the surface irradiance as the lower limit euphotic observed.
\end{abstract}

Key words: Photosynthetic efficiency, primary production, assimilation ratio, euphotic zone

Resumen.- Se realizaron muestreos durante diciembre 2005; mayo y agosto 2006; febrero, mayo y noviembre 2007 en Bahía de La Paz (Cuenca Alfonso). Se consideraron 7 niveles en la zona eufótica $\left(Z_{\text {eu }}\right)$, correspondientes al 100, 55, 33, 10, 3,1 y $0,1 \%$ de la irradiancia superficial. Se estimaron las razones de asimilación con incubaciones in situ y se observaron 2 patrones básicos, uno con máximos subsuperficiales durante febrero y mayo de 2007 y otro con tendencia a incrementar sus valores hacia el límite inferior de la $Z_{\text {eu }}$ (diciembre 2005; mayo y agosto 2006 y noviembre 2007). Los mínimos se observaron en diciembre 2005 con una variación de 0,16 a 2,35 $\mathrm{mg} \mathrm{C}(\mathrm{mg} \mathrm{Cla})^{-1} \mathrm{~h}^{-1}$. En febrero 2007 se registró alta

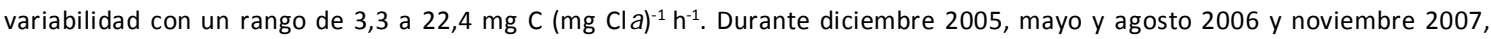

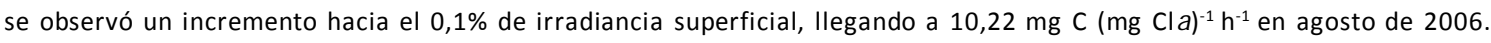
En mayo 2007, el máximo $\left(13,23 \mathrm{mg} \mathrm{C}\left(\mathrm{mg} \mathrm{Cla}^{-1} \mathrm{~h}^{-1}\right)\right.$ se asoció con la profundidad de la capa de mezcla. Al integrar la producción primaria (PP), se obtuvo un valor superior a los $350 \mathrm{~g} \mathrm{C} \mathrm{m}^{-2} \mathrm{año}^{-1}$, lo cual sugiere que la bahía es de carácter productivo alto. Se observó un incremento significativo en la PP al considerar el 0,1\% de la irradiancia superficial como el límite inferior de la zona eufótica.

Palabras clave: Eficiencia fotosintética, producción primaria, razón de asimilación, zona eufótica

\section{INTRODUCCIÓN}

Al igual que todos los ecosistemas, el sistema epipelágico del océano requiere de la entrada de carbono inorgánico para la síntesis de la materia orgánica a través de la fotosíntesis. En ésta transformación el fitoplancton tiene un papel primordial, ya que al ser consumido por el zooplancton y otros organismos planctónicos, los compuestos sintetizados son incorporados a la trama trófica marina. Esto implica además la transferencia o flujo de materia orgánica producida en la zona eufórica hacia zonas profundas del océano (Ducklowet al. 2001, BarocioLeón et al. 2007), al formar un vínculo entre el dominio planctónico y nectónico con el bentónico. A pesar de que el proceso fotosintético se genera principalmente en la zona eufótica $\left(\mathrm{Z}_{\mathrm{eu}}\right)$, tradicionalmente definida por la 
profundidad a la cual penetra el $1 \%$ de la irradiancia superficial $\left(\mathrm{E}_{0}\right)$, no debe pasarse por alto que por debajo de esta zona existe luz y que si bien no es la óptima para saturar este proceso, pudiera activarlo aunque con menor eficiencia, así como con tasas de crecimiento extremadamente lentas en la comunidad fitoplanctónica al ser contrastadas con las observadas en superficie (Andrea et al. 1998). Esta hipótesis no es nueva, Russell (1931) ya mencionaba que bajo la $Z_{\text {eu }}$ se encuentra una zona de penumbra o crepuscular en la cual se registra cierta irradiancia y que se puede extender en algunas regiones hasta cerca de $150 \mathrm{~m}$ de profundidad (Partensky et al. 1996). Para este caso bajo el criterio del límite al $0,1 \%$ Eo la zona de penumbra quedaría incluida en la zona eufótica. Además, es importante mencionar que bajo situaciones de limitación fotoquímica es posible la asimilación de carbono por rutas alternas no fotosintéticas, particularmente en ambientes con alta producción autótrofa y que presentan condiciones de mínimo oxigeno (Thomas 1963, Kremer 1978, Farías et al. 2009).

Uno de los atributos más importantes de documentar en los ecosistemas marinos es la variabilidad temporal y la magnitud de su producción primaria, así como identificar los factores que la regulan. Algunos parámetros como las razones de asimilación ( $\mathrm{PP} / \mathrm{Cl} a$ ), proporcionan información de la eficiencia fotosintética por unidad de clorofila, por lo cual han sido utilizados como indicador de la eficiencia en la producción primaria de comunidades fitoplanctónicas naturales (Gaxiola-Castro \& Álvarez-
Borrego 1986, Lara-Lara et al. 1993). En cierta forma este parámetro fotosintético es un indicador para estudiar uno de los cuestionamientos recurrentes en el medio oceánico que se refiere a determinar qué o cuál factor puede limitar la producción primaria. En la Bahía de La Paz, no existen registros de razones de asimilación del fitoplancton por lo que es de gran importancia el generar la información que permita inferir sobre la eficiencia fotosintética de los productores primarios.

En la Bahía de La Paz, los estudios relacionados con la producción primaria son escasos y no se habían realizado de manera sistemática. Durante los últimos 10 años se ha abordado este tema desde diferentes puntos de vista y mediante diversas técnicas, tales como las estimaciones mediante fluorescencia natural (Reyes-Salinas et al. 2003, Cervantes-Duarte et al. 2005, Verdugo-Díaz et al. 2008, Villegas-Aguilera 2009) y la determinación de los parámetros fotosintéticos de la comunidad fitoplanctónica (Verdugo-Díaz et al. 2012). Este estudio representa el primer intento de estimar las tasas de asimilación y la producción primaria integrada en la Bahía de La Paz, considerando que el límite inferior de la $\mathrm{Z}_{\mathrm{eu}}$ corresponde a la profundidad a la cual se registra el $0,1 \%$ de la irradiancia superficial.

\section{Materiales Y MÉTODOS}

La Bahía de La Paz, BCS., México, se localiza en la costa occidental del Golfo de California, entre $24^{\circ} 06^{\prime}-24^{\circ} 47^{\prime} \mathrm{N}$ y $110^{\circ} 18^{\prime}-110^{\circ} 45^{\prime} \mathrm{W}$ (Fig. 1) es el cuerpo de agua costero
Figura 1. Localización del área de estudio en Bahía de La Paz (Cuenca Alfonso), México / Location of the study area in La Paz Bay (Cuenca Alfonso), Mexico

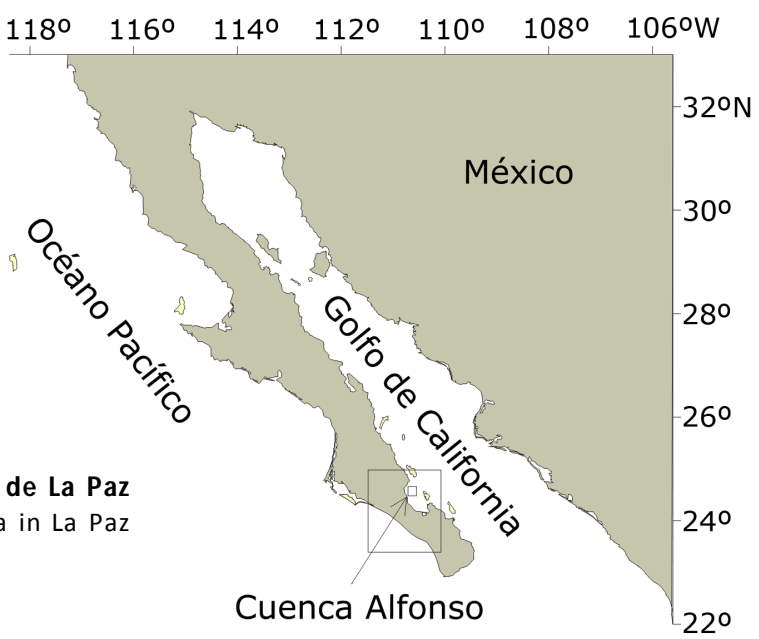


más grande y profundo del Golfo de California. Tiene dimensiones aproximadas de $80 \mathrm{~km}$ de largo y $35 \mathrm{~km}$ de ancho, está separado del golfo por el complejo insular Espíritu Santo. Contiene una depresión marina con umbral llamada Cuenca Alfonso ( $24^{\circ} 35^{`} \mathrm{~N}, 110^{\circ} 36^{\prime} \mathrm{W}$ ), en la cual se ha reportado un incremento gradual de la profundidad de sur a norte, con una pendiente aproximadamente del $1 \%$, hasta alcanzar la isobata de los $400 \mathrm{~m}$ en su límite norte (Anónimo 1982, Murillo-Jiménez 1987).

Se realizaron muestreos durante épocas contrastantes en la hidrografía de la zona, representadas por diciembre 2005, mayo y agosto 2006; y febrero, mayo y noviembre 2007 en una estación ubicada a $24^{\circ} 38$ N y $110^{\circ} 35^{`}$ W. Para obtener los datos de temperatura de la columna de agua se realizó un lance del perfilador de conductividad, temperatura y profundidad (CTD) a $100 \mathrm{~m}$ de profundidad. Así mismo, los datos generados por el CTD se utilizaron para calcular la profundidad de la capa de mezcla de acuerdo a lo propuesto por Kara et al. (2000). Se calculó la profundidad de la capa cuasi-isotérmica $\left(h_{\theta}(x, t)\right)$ y la profundidad de la capa cuasi-isohalina $(h s(x, t))$ usando el criterio $\Delta \theta$ para la temperatura potencial y el criterio $\Delta S$ para la salinidad $\left(h_{\theta}(x, t)\right)$.

Se tomaron muestras de la columna de agua a 7 niveles de irradiancia, correspondientes al 100, 55, 33, 10, 3, 1 y $0,1 \%$ de la irradiancia superficial (Eo). Los cálculos de profundidad correspondientes a cada nivel provienen de lecturas del disco de Secchi, según lo estipulado por Brown et al. (1989). La recolección de las muestras de agua de cada uno de los niveles considerados se llevó a cabo mediante una botella Niskin. La asimilación de carbono se determinó con la técnica del ${ }^{14} \mathrm{C}$ de Steemann Nielsen (1952). Para este fin se llevaron a cabo experimentos de incubación, inoculando cada muestra con $1 \mu \mathrm{Ci}$ de $\mathrm{NaH}^{14} \mathrm{CO}_{3}$ en botellas de policarbonato de $250 \mathrm{ml}$ de capacidad que se incubaron in situ durante una hora a la misma profundidad en que fueron recolectadas. En cada uno de los meses considerados, las incubaciones fueron realizadas entre las 11:00 y 13:00 h del tiempo local. Las emisiones $\beta$ se midieron en un contador de centelleo líquido Beckman LS6500 en el Centro de Investigación Científica y de Educación Superior de Ensenada Baja California (CICESE). Una vez obtenidos los valores de producción primaria (PP), según lo estipulado por Steeman-Nielsen 1952), se procedió a calcular las razones de asimilación $\left(P P^{B}=\frac{P P}{C l a}\right)$.
De manera simultánea a la recolecta de muestras para producción primaria se tomaron $4 \mathrm{~L}$ de agua para realizar determinaciones de concentración de Cla. Las muestras fueron depositadas en galones obscuros y mantenidas a baja temperatura durante el tiempo de transporte al laboratorio (aproximadamente $1 \mathrm{~h} 30 \mathrm{~min}$ ). El volumen filtrado de cada muestra fue el máximo posible según lo permitiera la concentración de partículas. Para este proceso se utilizaron filtros Whatman GF/F de $0,7 \mu \mathrm{m}$ de poro. Los procedimientos empleados en los análisis de clorofila $a$ y nitrógeno inorgánico disuelto $\left(\mathrm{NID}=\mathrm{NH}_{4}+\mathrm{NO}_{3}+\right.$ $\mathrm{NO}_{2}$ ), fueron los propuestos por Strickland \& Parsons (1972), utilizando un espectrofotómetro Lambda 25 (UV/VIS Spectrometer Perkin Elmer). La concentración del pigmento se obtuvo mediante las ecuaciones propuestas por Jeffrey \& Humphrey (1975). El amonio fue analizado inmediatamente después del muestreo, mientras que las muestras destinadas para determinar el NID fueron filtradas con filtros Whatman GF/F de $0,7 \mu \mathrm{m}$ de poro, congeladas y almacenadas a $-20^{\circ} \mathrm{C}$ para su posterior análisis.

Los valores de producción primaria y concentración de nutrientes obtenidos en los niveles de luz considerados, fueron integrados mediante el método trapezoidal, para así obtener un dato representativo de la zona eufótica para cada uno de los meses considerados. Para obtener la producción primaria diaria el valor originalmente obtenido $\left(\mathrm{mg} \mathrm{C} \mathrm{m} \mathrm{m}^{-2} \mathrm{~h}^{-1}\right.$ ) fue multiplicado por 13,5 que es el periodo de horas luz en la zona de estudio según la propuesta de Kirk (1994) y ValdezHolguín et al. (1995), y posteriormente extrapolado a los 365 días del año.

\section{Resultados}

La temperatura superficial presentó una marcada variabilidad estacional, registrando el valor mínimo $\left(19,7^{\circ} \mathrm{C}\right)$ durante febrero 2006 , en tanto que el valor máximo $\left(30^{\circ} \mathrm{C}\right)$ ocurrió en agosto del mismo año (Fig. 2). Se pudieron identificar meses asociados con temperaturas altas, mismos que se denominaron como meses cálidos (julio, agosto, septiembre y octubre) los cuales presentaron valores de temperatura superficial superiores a $28^{\circ} \mathrm{C}$. Los meses considerados fríos (diciembre 2005 y marzo 2006) mostraron temperaturas superficiales inferiores a $22^{\circ} \mathrm{C}$. Al contrastar los valores registrados de esta variable entre 2006 y 2007, se hace evidente que el primer año registró temperaturas mayores, sobre todo durante el período cálido cuando se observaron las 


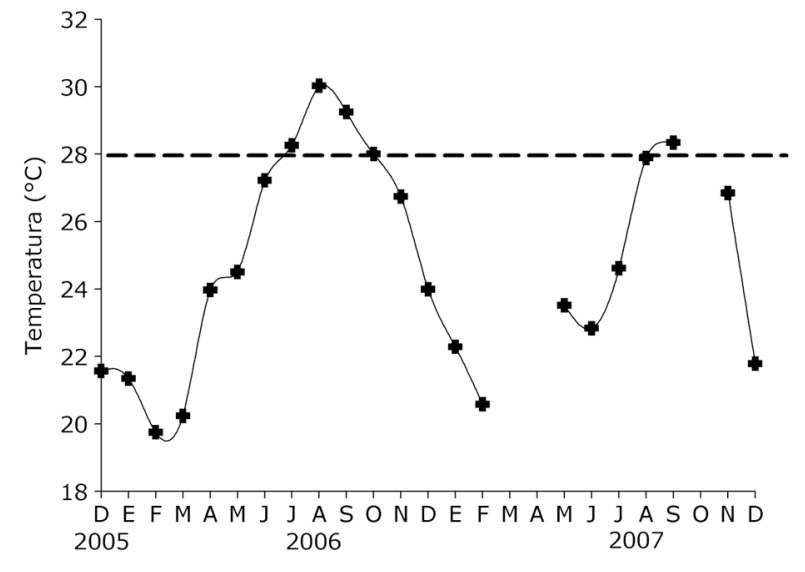

Figura 2. Variabilidad de la temperatura superficial de diciembre 2005 a junio 2007. La línea punteada indica la temperatura que se consideró para separar los meses cálidos de los meses fríos / Surface temperature variability December 2005 to June 2007. The dashed line indicates the temperature for separating the warm months of the cold months

mayores diferencias entre los meses de junio de los años considerados $\left(4,4^{\circ} \mathrm{C}\right)$.

Durante diciembre 2005 y febrero 2007 la temperatura fue relativamente homogénea en la columna de agua y la capa de mezcla se localizó en ambos casos por debajo de $90 \mathrm{~m}$. El límite inferior de la $\mathrm{Z}_{\mathrm{eu}}$ se registró dentro la capa de mezcla (65 y $82 \mathrm{~m}$ respectivamente). Por el contrario, tanto mayo y agosto 2006 como mayo y noviembre 2007 presentaron una columna de agua estratificada, con profundidades de la capa de mezcla inferiores a $25 \mathrm{~m}$. Bajo estas condiciones, la $\mathrm{Z}_{\text {eu }}$ fue siempre superior a la profundidad de la capa de mezcla al presentar profundidades mayores a $60 \mathrm{~m}$.

La distribución espacio-temporal de la concentración de clorofila evidenció la presencia de concentraciones altas en niveles subsuperficiales, principalmente en los registros de mayo de 2006 y 2007, alcanzando valores cercanos a 4,0 mg Cla m $\mathrm{m}^{-3}$, con máximas concentraciones

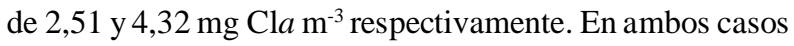
se localizaron por debajo de la capa de mezcla en donde se obtuvo aproximadamente el nivel del 3\% de la irradiancia superficial. Es importante señalar que el valor

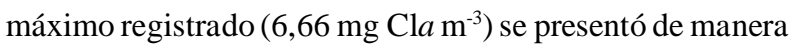
extraordinaria en el nivel superficial durante diciembre 2005 (Fig. 3) y que fue asociado a la proliferación de Myrionecta rubra (Villegas-Aguilera 2009).

De manera general, en la distribución del NID se observó un incremento con la profundidad (Fig. 4). Los valores mínimos se registraron en dentro de los niveles de 55 y $33 \%$ de la irradiancia superficial, mientras que los máximos coincidieron invariablemente en el límite inferior de la $\mathrm{Z}_{\text {eu }}$.

Los perfiles de las razones de asimilación $\left(\mathrm{PP}^{\mathrm{B}}\right)$ muestran que su distribución vertical presenta 2 patrones básicos; uno con máximos subsuperficiales (Figs. 5d, 5e) y otro con una clara tendencia a incrementar sus valores hacia el límite inferior de la $Z_{\text {eu }}$ (Fig. 5a, 5c y 5f). Los valores menores se observaron en diciembre 2005 con un rango de variación de 0,16 a 2,35 mg C (mg Cla $)^{-1} \mathrm{~h}^{-1}$. En tanto que en febrero 2007 se encontró el valor máximo en el 1\% de irradiancia superficial, además de una gran variabilidad en la distribución vertical de sus valores (3,3 a 22,4 mg C (mg Cla $)^{-1} \mathrm{~h}^{-1}$ (Fig. 5d). Durante diciembre 2005, mayo y agosto 2006 y noviembre 2007, se observó un incremento en la razón de asimilación en el $0,1 \%$ de irradiancia superficial (Figs. 5a, 5b, 5c y 5f), con un máximo de 10,22 $\mathrm{mg} \mathrm{C}(\mathrm{mg} \mathrm{Cl} a)^{-1} \mathrm{~h}^{-1}$ durante agosto (Fig. 5c). En el perfil de mayo 2007, el valor mayor de la razón de asimilación se registró en la profundidad de la capa de mezcla $(13,23 \mathrm{mg}$ $\mathrm{C}(\mathrm{mg} \mathrm{Cl} a)^{-1} \mathrm{~h}^{-1}$, observándose un decremento con la profundidad (Fig. 5e). Un aspecto sobresaliente de los perfiles de mayo en ambos años es el desarrollo de 2 incrementos en el perfil vertical, uno asociado a la capa de mezcla y otro en la base de la $Z_{\text {eu }}$ entre el 1 y $0,1 \%$ de $\mathrm{E}_{0}$.

Con relación a la producción primaria integrada en la zona eufótica se estimó un valor de $271,8 \mathrm{~g} \mathrm{C} \mathrm{m}^{-2} \mathrm{año}^{-1}$, considerando la definición tradicional del $1 \%$ de la irradiancia superficial como límite inferior de la $\mathrm{Z}_{\mathrm{eu}}$ y un valor de 406,2 $\mathrm{g} \mathrm{C} \mathrm{m}^{-2}$ año $^{-1}$, al extender la definición de ésta hasta la profundidad del $0,1 \%$.

En la presente investigación se consideró que el límite inferior de la $\mathrm{Z}_{\text {eu }}$ corresponde al $0,1 \%$ de la irradiancia superficial, a diferencias de otros autores que han asumido que este punto se encuentra a la profundidad en que la irradiancia superficial es igual al 1\%. De esta forma, se pudo observar que la región de la $\mathrm{Z}_{\mathrm{eu}}$ en donde los registros de irradiancia oscilan entre el 1 y $0,1 \%$ presenta valores entre los 23,8 y $33,3 \mathrm{mg} \mathrm{C} \mathrm{m}^{-3} \mathrm{~h}^{-1}$, mismos que representan de manera constante aproximadamente el 33\% de la producción primaria integrada (Fig. 6). Al respecto se realizó una comparación entre los valores mensuales de producción primaria en ambos niveles, obteniendo según la prueba no paramétrica de Wilcoxon que las diferencias observadas son significativas $(P<0,05)$. Al correlacionar el NID integrado con la producción primaria también integrada no se observó que los nutrientes fueran 
Figura 3. Distribución vertical de clorofila a (Cla) y temperatura durante diciembre 2005 (a), mayo 2006 (b), agosto 2006 (c), febrero 2007 (d), mayo 2007 (e) y noviembre 2007 (f). La línea punteada indica la profundidad de la capa de mezcla. Los puntos indican el porcentaje de irradiancia superficial de los 7 niveles / Vertical distribution of chlorophyll a (Chla) and temperatura during December 2005 (a), May 2006 (b), August 2006 (c), February 2007 (d), May 2007 (e) and November 2007 (f). The dotted line indicates the depth of the mixed layer. The dots indicate the percentage of surface irradiance of 7 levels

Figura 4. Distribución vertical del NID $(\mu \mathrm{M})$ durante diciembre 2005 (a), mayo 2006 (b), agosto 2006 (c), febrero 2007 (d), mayo 2007 (e) y noviembre 2007 (f) / Vertical distribution of NID (M) during December 2005 (a), May 2006 (b), August 2006 (c), February 2007 (d), May 2007 (e) and November 2007 (f)
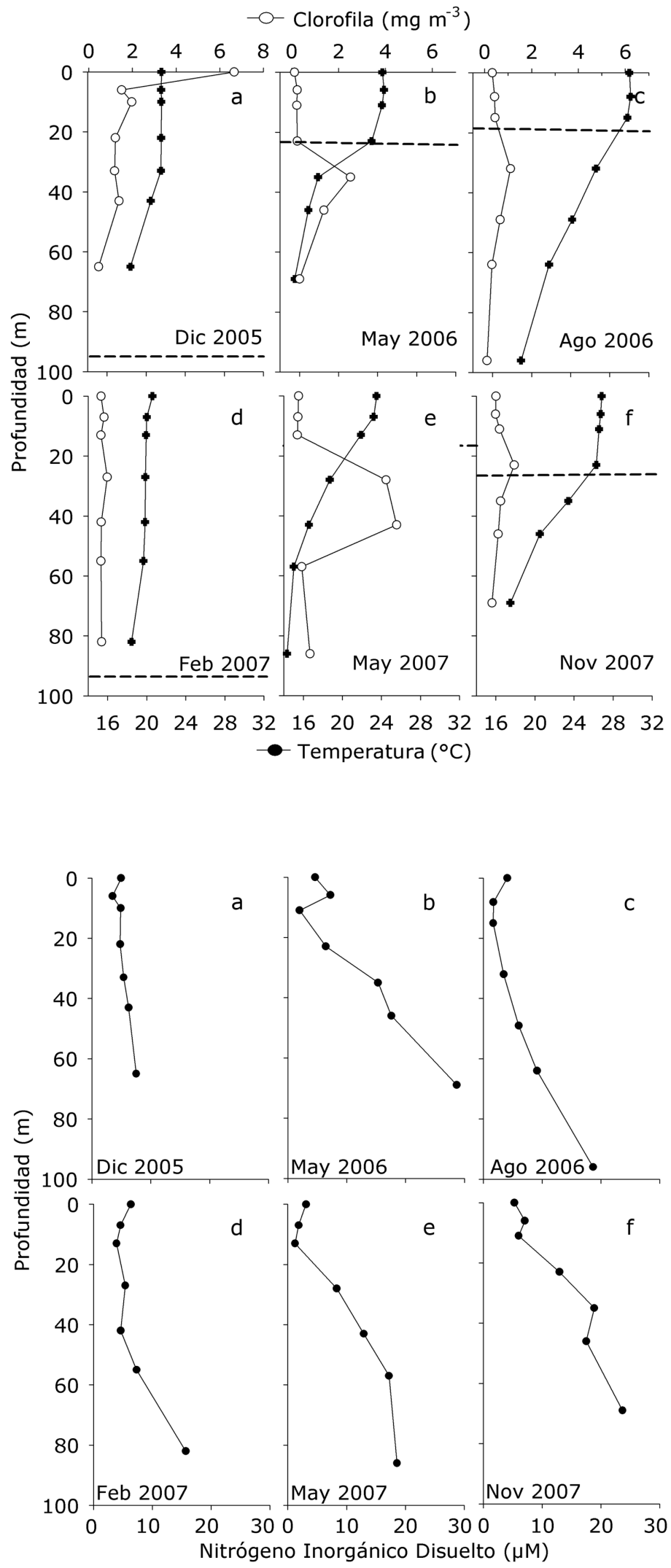

Vol. 49, № 3, 2014

Revista de Biología Marina y Oceanografía 
Figura 5. Distribución vertical de las razones de asimilación [ $\left.\mathrm{mg} \mathrm{C} \mathrm{(mg} \mathrm{Cla})^{-1} \mathrm{~h}^{-1}\right]$ durante diciembre 2005 (a), mayo 2006 (b), agosto 2006 (c), febrero 2007 (d), mayo 2007 (e) y noviembre 2007 (f) / Vertical distribution of assimilation ratios [mg C ( $\mathrm{mg} \mathrm{Chla})^{-1} \mathrm{~h}^{-1}$ ] during December 2005 (a), May 2006 (b), August 2006 (c), February 2007 (d), May 2007 (e) and November 2007 (f)
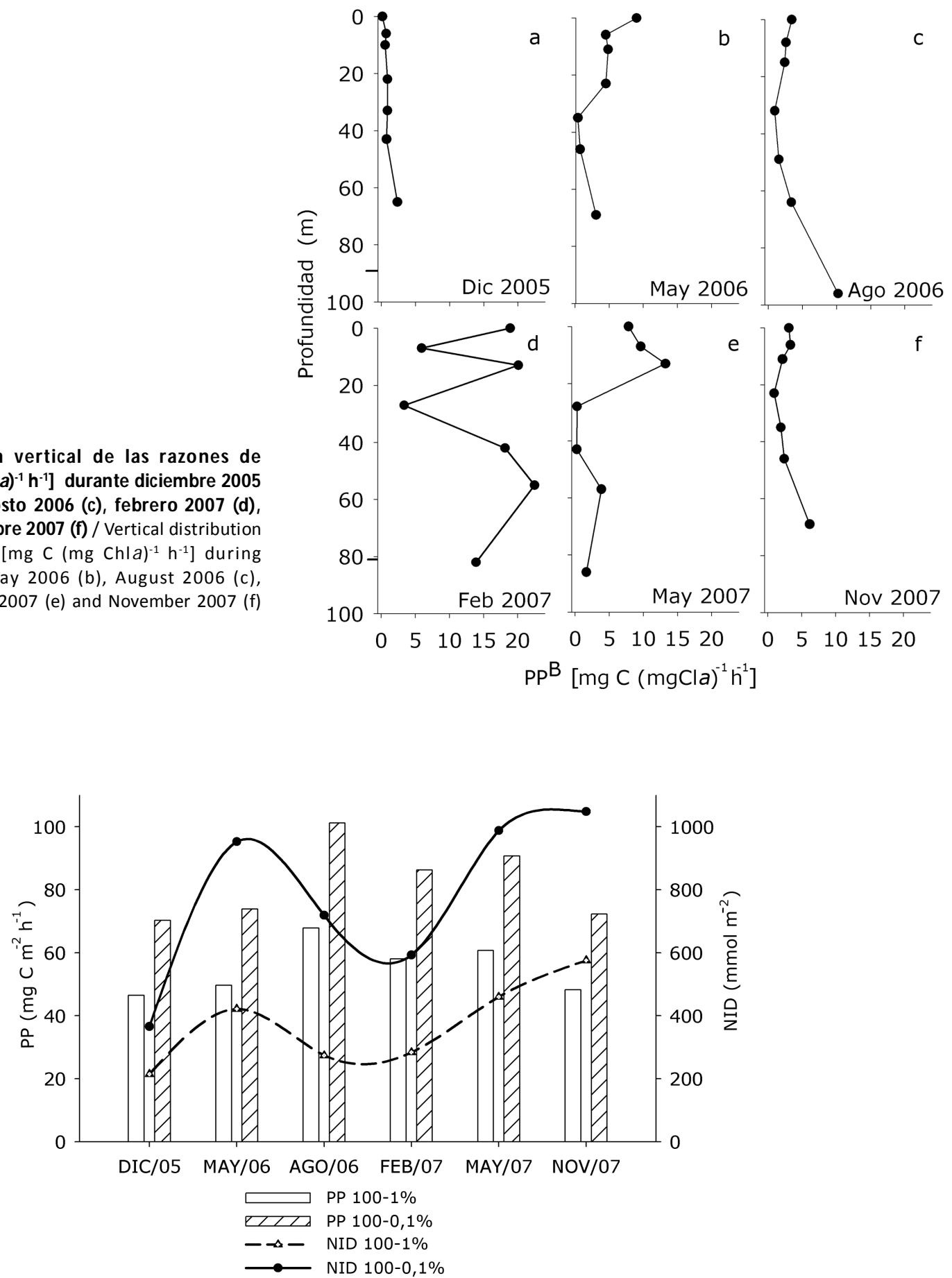

Figura 6. Producción primaria integrada y concentración de nitrógeno orgánico disuelto (NID) durante el periodo de estudio / Integrated primary production and dissolved inorganic nitrogen (NID) concentration during the study period 
el único factor regulador de la actividad fotosintética, dado que los meses en los cuales se determinó la mayor concentración de estos nutrientes no necesariamente fueron los que presentaron valores máximos de producción primaria, más bien, condiciones en las cuales los nutrientes presentaron valores intermedios, coinciden con los mayores datos de producción primaria integrada (agosto 2006). De igual manera, al aumentar la profundidad de la $\mathrm{Z}_{\text {eu }}$ del 1 al $0,1 \%$ de la irradiancia superficial, la concentración de nutrientes se incrementa considerablemente al registrar valores de aguas más profundas y de mayor concentración de nutrientes (generalmente $>18 \mu \mathrm{M}$ ).

\section{Discusión}

En la Bahía de La Paz, el rango de valores de las razones de asimilación registrado en la presente investigación (0,2-21 $\mathrm{mg} \mathrm{C}(\mathrm{mg} \mathrm{Cl} a)^{-1} \mathrm{~h}^{-1}$ fue superior al reportado para diferentes zonas del Golfo de California, dado que según GaxiolaCastro \& Álvarez-Borrego (1986), este varía entre 0,84 y $7,3 \mathrm{mg} \mathrm{C}(\mathrm{mg} \mathrm{Cl} a)^{-1} \mathrm{~h}^{-1}$. De igual manera, Lara-Lara et al. (1993) registraron variaciones entre 1,9 a $15 \mathrm{mg} \mathrm{C} \mathrm{(mg} \mathrm{Cla})^{-1}$ $\mathrm{h}^{-1}$ para la parte central y sur del golfo. Los valores más próximos a los aquí reportados son los determinados por Bustos-Serrano et al. (1996), con valores de 10 a $25 \mathrm{mg} \mathrm{C}$ $(\mathrm{mg} \mathrm{Cl} a)^{-1} \mathrm{~h}^{-1}$ y de 1 a $7,2 \mathrm{mg} \mathrm{C}(\mathrm{mg} \mathrm{Cl} a)^{-1} \mathrm{~h}^{-1}$ para una laguna costera del norte del Golfo de California. Sin embargo, en este caso es relevante discutir la distribución vertical de los valores discretos, particularmente para agosto 2006 y febrero 2007, ya que en estos meses se observó un incremento gradual hacia el $0,1 \%$ de la irradiancia superficial o límite inferior de la $\mathrm{Z}_{\mathrm{eu}}$. Esta distribución proporciona evidencia que a bajas irradiancias la fotoadaptación pigmentaria de las células juega un papel de suma importancia, ya que durante estos 2 meses se registraron los valores máximos de las razones de asimilación, no obstante que las concentraciones de nutrientes no fueron las más elevadas. Este proceso fisiológico es una respuesta a la mezcla vertical o al hundimiento de las células fitoplanctónicas que al encontrarse en condiciones de baja irradiancia tienen la capacidad de activar y saturar el proceso fotosintético en el fotosistema II, aun cuando la disponibilidad en el número de fotones es menor (Moore et al. 2006). Bajo estas condiciones se pueden desarrollar especies del género Prochlorococcus y de otros grupos del fitoplancton de tamaños pequeños (nano y picofitoplancton) reportados como responsables de hasta el 90\% de la producción primaria en el medio marino (Valiela 1995, Nona et al. 2000, Goeriecke et al. 2000) y que de manera particular para zonas aledañas a esta bahía pueden proliferar y sostener altos valores de producción primaria, incluso bajo condiciones de oligotrofia (Valdez-Holguín et al. 1995). Estrategias similares de fotoadaptación han sido observadas en el picofitoplancton de ambientes oligotróficos incluido el Pacífico Mexicano (Partensky et al. 1999, Almazán-Becerril \& García-Mendoza 2008) y también en estuarios en los cuales la atenuación de la luz es importante debido a la gran cantidad de material particulado (Pan et al. 2007).

La capacidad fotosintética no es una función directa de la absorción de fotones (Sakshaug et al. 1997) sino del umbral de saturación de sus mecanismos fotosintéticos. Las irradiancias observadas en el periodo de estudio en la base de la $Z_{\text {eu }}$ oscilaron entre los 0,6 y $1,1 \mu$ mol cuanta $\mathrm{m}^{-2}$ $\mathrm{s}^{-1}$. Al respecto se ha reportado que las comunidades aclimatadas a zonas de penumbra pueden saturar su proceso fotoquímico a irradiancias menores a $90 \mu \mathrm{mol}$ cuanta $\mathrm{m}^{-2} \mathrm{~s}^{-1}$ y que en algunas zonas específicas pueden saturar sus procesos incluso a irradiancias menores a 12 $\mu \mathrm{mol}$ cuanta $\mathrm{m}^{-2} \mathrm{~s}^{-1}$ (Oduor \& Schagerl 2007). Por el contrario, las células cercanas a la superficie requieren valores mayores a $300 \mu \mathrm{mol}$ cuanta $\mathrm{m}^{-2} \mathrm{~s}^{-1}$ (Verdugo-Díaz et al. 2012). Al respecto Prieto et al. (2008) mencionan la importancia de la interrelación de variables como irradiancia, abundancia celular y concentración de clorofila para la absorción de luz, y sobre todo de la eficiencia cuántica para la asimilación del carbono, como los factores de mayor peso para obtener tasas fotosintéticas mayores. De igual forma, existen poblaciones de cianofitas como Prochlorococcus, presentes en el sitio de estudio (Martínez-López, datos no publicados), en las cuales la clorofila $a$ no es el único ni el principal pigmento fotosintético, dado que presentan divinil clorofila $a$ como importante activador del proceso fotosintético (Moore et al. 1995, Claire et al. 2002, Everroad et al. 2006). Los resultados obtenidos y las observaciones de este género en el sitio de estudio, sugieren que estos microorganismos contribuyen de manera importante en el incremento en las razones fotosintéticas, de manera similar a lo reportado en otros sitios (Goericke et al. 2000).

En los meses en los cuales los perfiles de las razones de asimilación mostraron una mayor variabilidad (mayo 2006, febrero y mayo 2007), fue común observar un valor máximo subsuperficial asociado a la formación de la termoclina, ya que el cambio de densidad en esta zona actúa como una barrera física y favorece la acumulación de partículas y/o células fotosintéticas (Sharples et al. 2001, Verdugo-Díaz et al. 2012), mismas que de manera oportunista aprovechan las condiciones existentes 
incrementando su eficiencia fotosintética. Al respecto, López-Sandoval et al. (2009) mencionan que tanto la profundad de la termoclina como de la capa de mezcla son factores relevantes para las comunidades autótrofas al regular la disponibilidad de nutrientes así como la capacidad de penetración de la radiación fotosintéticamente activa que a su vez regulan la fotosíntesis y el crecimiento que son 2 de las funciones fisiológicas principales de la comunidad mencionada.

La estimación de valores de producción primaria integrada para el sitio de estudio, superiores a los $350 \mathrm{~g} \mathrm{C}$ $\mathrm{m}^{-2}$ año ${ }^{-1}$ dan la pauta para considerar a esta zona como de alta producción, considerando lo propuesto por Boynton et al. (1982). Esto es congruente con los registros en el Golfo de California publicados por GaxiolaCastro et al. (1995), que reportaron un máximo de $1861 \mathrm{~g}$ $\mathrm{C} \mathrm{m}^{-2} \mathrm{año}^{-1} \mathrm{o}$ los de Martínez-Gaxiola et al. (2007), que estimaron máximos cercanos a $365 \mathrm{~g} \mathrm{C} \mathrm{m}^{-2} \mathrm{año}^{-1}$. Incluso para la misma Bahía de La Paz ya se ha reportado un máximo de $824 \mathrm{~g} \mathrm{C} \mathrm{m}^{-2} \mathrm{año}^{-1}$ (Cervantes-Duarte et al. 2005).

Por otro lado, los valores de clorofila observados en este estudio son similares a reportes previos para la Bahía de La Paz (Lavaniegos \& López-Cortéz 1997, MartínezLópez et al. 2001, Reyes-Salinas et al. 2003). Los incrementos de diciembre 2005 y mayo 2007 dados por especies del microfitoplancton (Villegas-Aguilera 2009), denotan la ocurrencia de proliferaciones de fitoplancton en diferentes estratos de la columna de agua, cuyos valores superan el valor medio $\left(1 \mathrm{mg} \mathrm{m}^{-3}\right)$ para el sitio de estudio. Los máximos de clorofila ocurrieron alrededor de la nitriclina o en la superficie cuando la capa de mezcla fue mayor. Sin embargo, la predominancia de valores de clorofila cercanos al promedio se pueden explicar por la dominancia de fitoplancton de talla pequeña $<20 \mu \mathrm{M}$ (Villegas-Aguilera 2009). Su crecimiento en la capa de mezcla entre diciembre y mayo, explica los valores bajos de nitratos en la capa de mezcla. En tanto que los valores bajos encontrados en agosto 2006 denotan el ingreso de agua Tropical Superficial, la cual se caracteriza por ser oligotrófica (Villegas-Aguilera 2009).

Es importante mencionar que en la presente investigación se consideró como límite inferior de la zona eufótica la profundidad del 0,1 y no del $1 \%$ de la irradiancia superficial. Los valores estimados considerando uno u otro de los límites de la zona eufótica son significativamente diferentes $(P<0,05)$, ya que en esta profundidad de mínima luz hay una mayor área y volumen que amplían la zona o el hábitat de las comunidades fitoplanctónicas y por consiguiente propicia la asimilación del carbono. Por ende, los datos integrados de producción primaria se incrementan aproximadamente en $33 \%$. De acuerdo a estos resultados, se puede afirmar que para estudios de producción primaria en la zona de estudio es recomendable considerar la profundidad en la cual se registre al menos el $0,1 \%$ de la irradiancia superficial como profundidad de compensación, ya que a este nivel se cumple la premisa de Khanna et al. (2009), según la cual se define la $\mathrm{Z}_{\text {eu }}$ como aquella profundidad en la cual la luz aun disponible permite la activación del proceso fotosintético con la respectiva asimilación de carbono por parte del fitoplancton.

\section{Agradecimientos}

Agradecemos el apoyo recibido del Instituto Politécnico Nacional (Proyecto SIP 20131145). G.V.D y A.M.L. son apoyados como becarios COFAA (Comisión de Operación y Fomento de Actividades Académicas) y EDI del Instituto Politécnico Nacional de México. Al IPN y al CONACYT por la beca PIFI y de posgrado para MMVA. G.G.C. del CICESE, División de Oceanología, es miembro del Sistema Nacional de Investigadores (SNI-CONACYT).

\section{LITERATURA CITADA}

Andrea E, J Alpine \& E Cloern. 1988. Phytoplankton growth rates in a light-limited environment, San Francisco Bay. Marine Ecology Progress Series 44: 167-173.

Anónimo. 1982. Omega Chart No. 21120. Bahía Magdalena to La Paz. Defense Mapping Agency Hydrographic Topographic Center, Washington.

Barocio-León AO, R Millán-Núñez, E Santamaría del Ángel \& A González-Silvera. 2007. Producción primaria del fitoplancton de la zona eufórica del sistema de la Corriente de California estimada mediante imágenes del CZCS. Ciencias Marinas 33(1): 59-72.

Boynton WR, WM Kemp \& CW Keefe. 1982. A comparative analysis of nutrients and other factors influencing estuarine phytoplankton production. In: Kennedy VS (ed). Estuarine comparisons, pp. 69-90. Academic Press, New York.

Brown J, A Colling, D Park, J Phillips, D Rhothery \& J Wrigth. 1989. Light and sound in seawater. In: Bearman G (ed). Seawater: its composition, properties and behavior, pp. 60-85. The Open University, Milton Keynes.

Bustos-Serrano H, R Millán-Núñez \& R Cajal-Medrano. 1996. Efecto de la marea en la producción orgánica primaria en una laguna costera del Canal de Ballenas, Golfo de California. Ciencias Marinas 22(2): 215-233. 
Cervantes-Duarte R, G Verdugo-Díaz \& JE Valdez-Holguín. 2005. Modelo estacional de producción primaria estimada mediante fluorescencia natural en una región costera del Golfo de California, México. Hidrobiológica 15(1): 79-87.

Claire ST, R Gabrielle, K Jonathan \& WC Sallie. 2002. Cyanobacterial photosynthesis in the oceans: the origins and significance of divergent light-harvesting strategies. Trends in Microbiology 10(3): 134-142.

Ducklow HW, DK Steinberg \& KO Buesseler. 2001. Upper ocean carbon export and the biological pump. Oceanography 14(4): 50-58.

Everroad C, C Six, F Partensky, JC Thomas, J Holtzendorff \& AM Wood. 2006. Biochemical bases of type IV chromatic adaptation in marine Synechococcus spp. Journal of Bacteriology 188(9): 3345-3356.

Farías 1, C Fernández, J Faundez, M Cornejo \& ME Alcaman. 2009. Chemolithoautotrophic production mediating the cycling of the greenhouse gases $\mathrm{N}_{2} \mathrm{O}$ and $\mathrm{CH}_{4}$ in an upwelling ecosystem. Biogeosciences 6: 3053-3069.

Gaxiola-Castro G \& S Álvarez-Borrego. 1986. Photosynthesisirradiance relationship for winter phytoplankton in Pacific waters off México. Oceanologica Acta 9: 497-501.

Gaxiola-Castro G, J García-Cordova, JE Valdez-Holguín \& M Botello-Ruvalcaba. 1995. Spatial distribution of chlorophyll $a$ and primary productivity in relation to winter physical structure in the Gulf of California. Continental Shelf Research 15: 1043-1059.

Goericke R, RJ Olson \& A Shalapyonok. 2000. A novel niche for Prochlorococcus sp. in low-light suboxic environments in the Arabian Sea and the Eastern Tropical North Pacific. Deep Sea Research Part I 47(7): 1183-1205.

Jeffrey SW \& GF Humphrey. 1975. New spectrophotometric equations for the determining chlorophylls $\mathrm{a}, \mathrm{b}, \mathrm{c} 1$ and $\mathrm{c} 2$ in higher plants, algae and natural phytoplankton. Biochemie und Physiologie der Pflanzen 167: 191-194.

Kara AB, P Rochford \& H Hurlburt. 2000. An optimal definition for ocean mixed layer depth, Journal. Geophysical Research 105(C7): 16803-16821.

Khanna DR, R Bhutiani \& KS Chandra. 2009. Effect of the euphotic depth and mixing depth on phytoplanktonic growth mechanism. Environmental Research 3(2):223-228.

Kirk JTO. 1994. Light y photosynthesis in aquatic ecosystems, 509 pp. Cambridge University Press, Cambridge.

Kremer BP. 1978. Determination of photosynthetic rates and ${ }^{14} \mathrm{C}$ photoassimilatory products de brown seaweeds. In: Hellebust JA \& JS Craige (eds). Handbooks of physiological methods: Physiological and biochemical methods, pp. 269283. Cambridge University Press, Cambridge.

Lara-Lara JR, R Millán-Núñez, JL Lara-Osorio \& C Bazán-Guzmán. 1993. Producción y biomasa del fitoplancton por clases de tamaño, en la parte central del Golfo de California durante primavera de 1985. Ciencias Marinas 19(2): 197-154.
Lavaniegos BE \& D López-Cortés. 1997. Fatty acid composition and community structure of plankton from the San Lorenzo Channel, Gulf of California. Estuarine Coastal and Shelf Science 45: 845-854.

López-Sandoval DC, JR Lara-Lara, MF Lavín, S ÁlvarezBorrego \& G Gaxiola-Castro. 2009. Primary productivity in the eastern tropical Pacific off Cabo Corrientes, México. Ciencias Marinas 35(2): 169-182.

Martínez-Gaxiola MD, R Sosa-Avalos \& G Gaxiola-Castro. 2007. Producción de carbono orgánico en los mares mexicanos (1998-2005). En: Hernández de la Torre B \& G Gaxiola-Castro (eds). Carbono en ecosistemas acuáticos de México, pp. 251-266. Secretaría del Medio Ambiente y Recursos Naturales, Instituto Nacional de Ecología, Centro de Investigación Científica y de Educación Superior de Ensenada, México.

Martínez-López A, R Cervantes-Duarte, A Reyes-Salinas \& JE Valdez-Holguín. 2001. Cambio estacional de clorofila $a$ en la Bahía de La Paz, B.C.S., México. Hidrobiológica 11: 45-42.

Moore CM, DJ Suggett, AE Hickman, YN Kim \& JF Tweddle, J Sharples, RJ Geider \& PM Holligan. 2006. Phytoplankton photoacclimation and photoadaptation in response to environmental gradients in a shelf sea. Limnology and Oceanography 51(2): 936-949.

Moore LR, R Goericke \& SW Chisholm. 1995. Comparative physiology of Synechococcus and Prochlorococcus: influence of light and temperature on growth pigments, fluorescence and absorptive properties. Marine Ecology Progress Series 116: 259-275.

Murillo-Jiménez J. 1987. Algunas características paleoceanográficas y cuerpos de agua inferidos a partir del registro paleontológico (Radiolaria) en laBahía de La Paz, BCS, México. Tesis Profesional, Departamento de Geología, Universidad Autónoma de Baja California Sur, La Paz, 70 pp.

Nona S, R Agawin, CM Duarte \& S Agustí. 2000. Nutrient and temperature control of the contribution of picoplankton to phytoplankton biomass and production. Limnology and Oceanography 45(3): 591-600.

Oduor SO \& M Schagerl. 2007. Phytoplankton primary productivity characteristics in response to photosinthetically active radiation in three Kenyan Rift Valley saline-alkaline lakes. Journal of Plankton Research 29(12): 1041-1050.

Pan LA, J Zhang \& LH Zhang. 2007. Picophytoplankton, nanophytoplankton, heterotrophic bacteria and viruses in the Changjiang Estuary and adjacent coastal waters. Journal of Plankton Research 29(2): 187-197.

Partensky F, WR Hess \& D Vaulot. 1999. Prochlorococcus, a marine photosynthetic prokaryote of global significance. Microbiology and Molecular Biology Reviews 63: 106-127. 
Partensky F, N Hoepner, WKW Li, O Ulloa \& D Vaulot. 1993. Photoacclimation of Prochlorococcus sp. (Prochlorophyta) stains isolated from the North Atlantic and the Mediterranean Sea. Plant Physiology 60: 285-296.

Prieto L, RD Vaillancourt, B Hales \& J Marra. 2008. On the relationship between carbon fixation efficiency and biooptical characteristics of phytoplankton. Journal of Plankton Research 30(1): 43-56.

Reyes-Salinas A, R Cervantes-Duarte, RA Morales-Pérez \& JE Valdez-Holguín. 2003. Variabilidad estacional de la producción primaria y su relación con la estratificación vertical en la Bahía de La Paz, B.C.S. Hidrobiológica 13(2): 103-110.

Russell FS. 1931. The vertical distribution of marine macroplankton. X. Notes on the behaviour of Sagitta in the Plymouth area. Journal of the Marine Biological Association of the United Kingdom 17: 391-414.

Sakshaug E, A Bricaud, Y Dandonneau, PG Falkowski, DA Kiefer, L Legendre, A Morel, J Parslow \& M Takahashi. 1997. Parameters of photosynthesis: definitions, theory and interpretation of results. Journal of Plankton Research 19: $1637-1670$.

Sharples J, CM Moore, TP Rippeth, PM Holligan, DJ Hydes, NR Fisher \& H Simpson. 2001. Phytoplankton distribution and survival in the thermocline. Limnology and Oceanography 46(3): 486-496.

Steemann-Nielsen E. 1952. The use of radio-active carbon $\left({ }^{14} \mathrm{C}\right)$ for measuring organic production in the sea. Journal du Conseil 18: 117-140.
Strickland JD \& TR Parsons. 1972. A practical handbook of seawater analysis. Fisheries Research Board of Canada, Bulletin 167: 1-311.

Thomas WH. 1963. Physiological factor affecting the interpretation of phytoplankton production measurements. In: Doty MS (eds). Proceedings of the Conference on Primary Productivity Measurements, Marine and Fresh Water, pp. 147-162. USA Atomic Energy Commission, Washington.

Valdez-Holguín JE, G Gaxiola-Castro \& R CervantesDuarte. 1995. Producción primaria en el Golfo de California, calculada a partir de la relación entre irradiancia superficial y clorofila de la zona eufótica. Ciencias Marinas 21(3): 311-329.

Valiela I. 1995. Marine ecological processes, 546 pp. SpringerVerlag, New York.

Verdugo-Díaz G, MO Albañez-Lucero \& R CervantesDuarte. 2008. Estimación de la producción primaria durante otoño-invierno en la Bahía de La Paz, B.C.S. México. Oceánides 23(1/2): 39-43.

Verdugo-Díaz G, A Martínez-López, G Gaxiola-Castro \& JE Valdez-Holguín. 2012. Phytoplankton photosynthetic parameters from the Gulf of California southern region. Revista de Biología Marina y Oceanografía 47(3): 527-535.

Villegas-Aguilera MM. 2009. Fitoplancton silíceo de la zona eufótica, como señal de la producción primaria en Cuenca Alfonso, Golfo de California. Tesis de Maestría, Centro Interdisciplinario de Ciencias Marinas, La Paz, BCS, México, 95 pp.

Recibido el 13 de noviembre de 2013 y aceptado el 4 de septiembre 2014

Editor: Claudia Bustos D. 